Pacific Journal of Mathematics

CONJUGATE SURFACES FOR MULTIPLE INTEGRAL
PROBLEMS IN THE CALCULUS OF VARIATIONS 


\section{CONJUGATE SURFACES FOR MULTIPLE INTEGRAL PROBLEMS IN THE CALCULUS OF VARIATIONS}

\section{R. DenNemeyer}

The Jacobi equation of the second variation for a multiple integral problem in the calculus of variations is a linear second order elliptic type partial differential equation provided certain hypotheses hold in the multiple integral problem. By means of the theory of quadratic forms in Hilbert space already present in the literature pertinent properties of solutions of such partial differential equations can be established. Here the pertinent property discussed is the vanishing of a solution on the boundary of a region, i.e. the existence of a conjugate surface of the differential equation. After developing the notion of focal point and stating the index theorems of the associated quadratic form, the existence of one parameter families of conjugate surfaces is shown, and illustrations of the theory are given.

1. Introduction. Fundamental theorems for quadratic forms in Hilbert space which are pertinent to problems in the calculus of variations are established in [7], [9] by Hestenes. Included in [7] is a theory of indices for an important class of quadratic forms arising in variational theory, and a general theory of focal points applicable to simple or multiple integral problems. Illustrations of the applications of focal point theory to one independent variable variational problems are given, as well as to boundary value problems for ordinary differential equations. The theory is, however, also applicable to multiple integral problems, and to boundary value problems for elliptic partial differential equations (indeed for integro-differential equations), and the author had this in mind in the formulation of the theory. In [9] there are general theorems on properties of quadratic forms applicable to variational problems involving functionals defined on classes of vector valued functions of $m$ independent variables and with higher order derivatives. These theorems have as consequences further theorems on properties of systems of partial differential equations, and existence and differentiability theorems are established.

The purpose here is to set down an extension of the highly developed theory of conjugate points for simple integral problems in the calculus of variations to multiple integral problems. The extension is afforded by the theory established in [7]. Here the multiple integral problems in mind are those where the integrand involves at most first order partial derivatives of a real valued function of $m$ real variables. The Jacobi equation of the second variation is then a linear second 
order partial differential equation, and it is relative to this equation that the notion of conjugate surface is considered.

For consistency all definition, terminology, and notation conventions used (apart from certain minor differences explicitly written down) are carried over from [7] and [9]. For brevity this material is not repeated, except where necessary for readability.

2. Hilbert space and subspaces. In the sequel $m$ is a fixed positive integer, and $\Omega$ denotes $m$ dimensional real euclidean space. Points of $\Omega$ are written $t=\left(t_{1}, \cdots, t_{m}\right), s=\left(s_{1}, \cdots, s_{m}\right), \cdots$ and $|t|$ is the usual length in $\Omega$. If $S$ is a subset of $\Omega$, then $I(S), S^{*}, \bar{S}$ and $c(S)$, mean, respectively, the interior of $S$, the boundary of $S$, the closure of $S$, and the complement of $S$. An interval $a_{k} \leqq t_{k} \leqq b_{k}, k=$ $1, \cdots, m$, is abbreviated $[a, b]$, and similarly for $(a, b)$. A region is a bounded open connected subset of $\Omega$. Letters such as $x, y, z, u, v$ are used for real valued functions defined on subsets of $\Omega$. The summation convention for repeated indices in a product is adopted, and all summations are from 1 to $m$. The subscripts $i, j, k$ always have the range $1, \cdots, m$, while the subscripts $p, q$ have the range $1,2, \cdots$. Subscripts $k, p, q$ are never used as summation indices. A partial derivative $\partial x / \partial t_{k}$ is often written $\dot{x}_{k}$.

Let $T$ be a fixed region of class $B^{1}$ (see [9], [10], [11]). Simple examples are: the interior of a sphere or interval in $\Omega$, the interior of the union of a finite number of closed contiguous nonoverlapping intervals. Also, the image of one of these regions under a continuous one-to-one transformation, which is such that the transformation and its inverse satisfy a uniform Lipschitz condition on every compact subset of their respective domains, is also a region of class $B^{1}$ (henceforth the superscript 1 is omitted).

The basic Hilbert space $\mathscr{P}$ is the class of functions $x$ of class $D^{(1)}$ on $T$ ([9], [10], [11]), which together with their first partial derivatives are square integrable on $T$. This is the space $\mathscr{Z}_{1}$ of [9], except that the functions are real scalar valued. A function $x \in \mathscr{H}$ need not be continuous on $T$, and is characterized by the following properties:

(i ) $x$ is essentially absolutely continuous on $T$ in the sense of Calkin and Morrey ([3], [10], [11]);

(ii) $x$ and the derivatives $\dot{x}_{k}, k=1, \cdots, m$, are square integrable on $T$;

$x$ is normalized by taking $x(t)=\lim _{h \rightarrow 0} x^{h}(t)$ for each $t \in T$ for which the limit exists, and $x(t)=0$ elsewhere. Here $x^{h}$ denotes the $h$-average of $x$ ([9], p. 314). This normalization of members of $\mathscr{C}$ is convenient in the sequel. The space $\mathscr{H}$ is also called a Sobolev space ([11], p. 19). The inner product on $\mathscr{H}$ is 


$$
(x, y)=\int_{T} \dot{x}_{j}(t) \dot{y}_{j}(t) d t+\int_{T} x(t) y(t) d t
$$

and the norm is

$$
\|x\|=(x, x)^{1 / 2} \text {. }
$$

An important subspace of $\mathscr{H}$ is the class of $x \in \mathscr{P}$ which "vanish" on the boundary $T^{*}$. More exactly, let $C_{0}^{\infty}$ denote the subclass consisting of all functions $x$ having continuous partial derivatives of all orders on $T$ and whose support set (closure of the set of points $t$ such that $x(t) \neq 0$ ) is contained in $T$. Then the subspace of interest is $\mathscr{A}=\overline{C_{0}^{\infty}}$, the closure under the norm in Eq. (2-2). This subspace is denoted by $\mathscr{K}_{10}$ in [9]. It can also be shown that $\mathscr{A}=\overline{\mathscr{K}}_{0}$, where $\mathscr{K}_{0}$ denotes the class of all Lipschitzian functions having support set contained in $T$. If $S$ is any subset of $\Omega$ for which the function classes are defined, then it will be convenient at times to write $\mathscr{C}(S), \mathscr{A}(S)$, $C_{0}^{\infty}(S)$, etc. For example, $C_{0}^{p}(S)$ denotes the class of all functions whose derivatives of order $\leqq p$ are continuous on $T$ and whose support set is contained in $S$.

In the sequel the following alternate characterization of $\mathscr{A}$ is useful. Consider the space $\mathscr{\mathscr { C }}=\mathscr{H}(T)$. Extend each $x \in \mathscr{C}$ to $\Omega$ by setting $x(t)=0$ for $t \in c(T)$. A function $x$ so extended need not belong to the Hilbert space $\mathscr{C}(\Omega)$. However the class of functions which do belong to $\mathscr{C}(\Omega)$ when so extended constitute the subspace $\mathscr{A}$.

3. Divergence theorem. Use is made in the sequel of the following extension of the divergence theorem established by Hestenes. See also Morrey [12], and Carson ([4]).

THEOREM 3.1. Let $S$ be a nonempty open set in $\Omega$, and let $M, N_{1}, \cdots, N_{m}$ be given integrable functions on $S$. Consider the linear functional

$$
L(x)=\int_{S}\left[M(t) x(t)+N_{j}(t) \dot{x}_{j}(t)\right] d t
$$

on various linear manifolds. The following statements are equivalent.

(a) $L(x)=0$ on $\mathscr{K}_{0}(S)$.

(b) $L(x)=0$ on $C_{0}^{p}(S), p$ a given positive integer.

( c) $L(x)=0$ on $C_{0}^{\infty}(S)$.

(d) Let $\varepsilon>0$ be given. Then $L(x)=0$ on $\mathscr{K}_{\varepsilon}(S)$, the class of all $x \in \mathscr{K}_{0}(S)$ whose support set has diameter less than $\varepsilon$.

(e) If $k$ is a given integer in the range $1, \cdots, m$, and $S_{k}$ denotes the projection of $S$ onto the $t_{k}$ axis, then there exists a set $Z_{k}$ 
of linear measure zero in $S_{k}$ such that for each interval $[a, b]$ in $S$ having neither $a_{k}$ nor $b_{k}$ in $Z_{k}$ the relation

$$
\sum_{j=1}^{m} \int_{a_{j}^{\prime}}^{b_{j}^{\prime}}\left[N\left(b_{j}, t^{\prime}\right)-N\left(a_{j}, t_{j}^{\prime}\right)\right] d t_{j}^{\prime}=\int_{a}^{b} M(t) d t
$$

holds (where primes denote the remaining $m-1$ coordinates, e.g.,

$$
\left(b, t_{j}^{\prime}\right)=\left(t_{1}, \cdots, t_{j-1}, b, t_{j+1}, \cdots, t_{m}\right),
$$

and

$$
\left.d t_{j}^{\prime}=d t_{1} \cdots d t_{j-1} d t_{j+1} \cdots d t_{m}\right) .
$$

In fact, for almost all intervals or spheres $R$ such that $\bar{R}$ is contained in $S$,

$$
\int_{R^{*}} N_{i}(\sigma) l_{i} d \sigma=\int_{R} M(t) d t
$$

holds, where $l_{i}, i=1, \cdots, m$, are the direction cosines of the outer normal to $R^{*}$ and $d \sigma$ donotes the surface element on $R^{*}$.

If $M$ is continuous and each $N_{k}$ has continuous first partial derivatives on $S$, and one of the statements (a)-(e) holds, then Eq. (3.2) holds for each sphere $R$ such that $\bar{R}$ is contained in $S$, and Eqs. (3.1) and (3.2) are each equivalent to

$$
\sum_{j=1}^{m} \frac{\partial N_{j}}{\partial t_{j}}=M(t)
$$

holding in $S$.

4. The quadratic form. Let $P, Q_{1}, \cdots, Q_{m}, R_{i j}, i, j=1, \cdots, m$ be given integrable functions on $T$. It is assumed that $R_{j i}(t)=R_{i j}(t)$, $t \in T$, for all $i, j=1, \cdots, m$. Then

$$
J(x)=\int_{T}\left\{P(t) x^{2}(t)+\left[2 Q_{i}(t) \dot{x}_{i}(t)\right] x(t)+R_{i j}(t) \dot{x}_{i}(t) \dot{x}_{j}(t)\right\} d t
$$

defines a quadratic form $J$ on $\mathscr{H}$. The associated bilinear form is

$$
J(x, y)=\int_{T}\left\{P x y+Q_{i}\left(x \dot{y}_{i}+\dot{x}_{i} y\right)+R_{i j} \dot{x}_{i} \dot{y}_{j}\right\} d t .
$$

Let

$$
\omega(t, x, \dot{x})=\frac{1}{2}\left(P x^{2}+2 Q_{i} \dot{x}_{i} x+R_{i j} \dot{x}_{i} \dot{x}_{j}\right) .
$$

Then 


$$
\begin{aligned}
J(x, y) & =\int_{T}\left(\omega_{x} y+\omega_{\dot{x}_{i}} \dot{y}_{i}\right) d t \\
& =\int_{T}\left(\omega_{y} x+\omega_{\dot{y}_{i}} \dot{x}_{i}\right) d t=J(y, x)
\end{aligned}
$$

for each $x \in \mathscr{H}, y \in \mathscr{X}$. The quadratic form $J$ can be written

$$
J(x)=K(x)+R(x)
$$

where

$$
\begin{gathered}
K(x)=\int_{T}\left\{P(t) x^{2}(t)+\left[2 Q_{i}(t) \dot{x}_{i}(t)\right] x(t)\right\} d t \\
R(x)=\int_{T} R_{i j}(t) \dot{x}_{i}(t) \dot{x}_{j}(t) d t
\end{gathered}
$$

are quadratic forms on $\mathscr{l}$.

It is further assumed that $P, Q_{1}, \cdots, Q_{m}$ are bounded on $T$. Then (Theorem 5.1, [7]), $K$ is $w$-continuous on $\mathscr{C}$. Further, thefunctions $R_{i j}$ are assumed continuous on $\bar{T}$, with the strong Legendre condition

$$
R_{i j}(t) \xi_{i} \xi_{j}>0 \quad t \in \bar{T}
$$

holding for each $m$-tuple $\xi \neq(0, \cdots, 0)$. Accordingly $R$ is positive definite on $\mathscr{A}$ and hence (Theorem 8.1, [9]), the quadratic form $J$ is a Legendre form on $\mathscr{A}$.

Let $\mathscr{B}$ be a linear manifold in $\mathscr{H}$. A function $x \in \mathscr{H}$ is said to be $J$-orthogonal to $\mathscr{B}$ if, for every $y \in \mathscr{B}, J(x, y)=0$. The set of all such $x$ is called the $J$-orthogonal complement of $\mathscr{B}$, denoted by $\mathscr{B}^{J}$. There may exist one or more $x \in \mathscr{B}$ which are $J$-orthogonal to $\mathscr{B}$, i.e., $x \in \mathscr{B} \cap \mathscr{B}^{j}$. A function $x$ having this property is called a $J$ null vector of $\mathscr{B}$. The set of $J$-null vectors of $\mathscr{B}$ is denoted by $\mathscr{B}_{0}$. Observe $J(x)=0$ on $\mathscr{B}_{0}$. The nullity of $J$ on $\mathscr{B}$ is the dimension of the submanifold $\mathscr{B}_{0}$ of $\mathscr{B}$. The index of $J$ on $\mathscr{B}$ is the dimension of the maximal linear submanifold $\mathscr{B}$ on which $J(x)<0$. The following basic theorem is proven in [7].

THeOREM 4.1. $J$ is of finite index and nullity on $\mathscr{A}$. If $\mathscr{B}$ is a subspace of $\mathscr{A}, L(x)$ a linear form (functional) on $\mathscr{B}$ such that $L(x)=0$ on the submanifold of $J$-null vectors of $\mathscr{B}$, then there exists a function $y \in \mathscr{B}$ such that $L(x)=J(x, y)$ on $\mathscr{B}$. The fuction $y$ can be chosen orthogonal to the submanifold of J-null vectors of $\mathscr{B}$, and if so chosen is unique.

5. Extremals of $J$. Let $x$ be a function in $\mathscr{H}$ such that

$$
J(x, y)=\int_{T}\left(\omega_{x} y+\omega_{\dot{x}_{i}} \dot{y}_{i}\right) d t=0
$$


holds for every $y \in \mathscr{A}$, i.e., $x$ is $J$-orthogonal to the subspace $\mathscr{A}$. From the Divergence Theorem and the fact that $C_{0}^{\infty}(T)$ is dense in $\mathscr{A}$ it follows that

$$
\int_{R^{*}} \omega_{\dot{x}_{i}} l_{i} d \sigma=\int_{R} \omega_{x} d t
$$

holds for almost all intervals and spheres $R$ whose closure lies in $T$. Eq. (5.1) is the Euler equation for the functional $J$ on $\mathscr{K}$. A function $x \in \mathscr{H}$ such that Eq. (5.1) holds for almost all intervals and spheres with the stated property is called an extremal of $J$.

Accordingly the linear manifold of extremals of $J$ is just $\mathscr{A}^{J}$, the $J$-orthogonal complement of $\mathscr{A}$. The submanifold $\mathscr{A}_{0}$ of $J$-null vectors of $\mathscr{A}$ is the class of extremals of $J$ which vanish on $T^{*}$. In view of Theorem 4.1 this submanifold is finite dimensional.

With additional hypotheses on the coefficients the usual differential equation characterization of extremals is obtained. First, if the functions $R_{i j}$ and $Q_{i}$ are of class $C^{1}(T)$ and $P \in C(T)$, and if it is known that the extremal $x$ is of class $C^{2}(T)$, then the Euler equation (5.1) is equivalent to

$$
E(x)=\frac{\partial}{\partial t_{i}}\left(R_{i j} \frac{\partial x}{\partial t_{j}}\right)-x\left(P-\sum_{i=1}^{m} \frac{\partial Q_{i}}{\partial t_{i}}\right)=0
$$

holding in T. Eq. (5.2) is the Euler equation associated with $J$ as usually written. The differential operator $E$ appearing in Eq. (5.2) may be referred to as the Euler operator. Under the preceding hypotheses it is an elliptic operator.

In order to insure that the extremal $x \in \mathscr{C}$ is of class $C^{2}(T)$ still further hypotheses are placed on the coefficients. The following is a special case of Sobolev's theorem as given by Friedrichs ([6]). Let $p$ be an integer, $p>m / 2+2$. In addition to the previous requirements assume the functions $R_{i j}$ and $Q_{i}$ are of class $C^{p}(T)$, and let $P \in C^{p-1}(T)$. Then if $x \in \mathscr{C}$ is an extremal, $x \in C^{2}(T)$. Henceforth it is assumed that the coefficients satisfy these additional requirements. Thus $x$ is an extremal of $J$ on $T$ if, and only if, $x \in \mathscr{C} \cap C^{2}(T)$ and Eq. (5.2) holds on T. From Theorem 4.1 it follows that there are at most a finite number of linearly independent solutions of the Euler equation (5.2) which vanish on the boundary. The dimension of the submanifold of such solutions of (5.2) is the nullity of the form $J$ on $\mathscr{A}$.

6. Focal points of $J$. Index theorems. In order to apply the theory of indices given in [7] there is considered in subsequent sections a one parameter family $\{\mathscr{A}(\lambda)\}$ of subspaces of $\mathscr{A}$, where the real parameter $\lambda$ is restricted to an interval $\lambda^{\prime} \leqq \lambda \leqq \lambda^{\prime \prime}$. The family 
$\{\mathscr{A}(\lambda)\}$ has the following properties:

(a) $\mathscr{A}\left(\lambda^{\prime}\right)$ has as its sole member the function which is zero everywhere in $T$, and $\mathscr{A}\left(\lambda^{\prime \prime}\right)=\mathscr{A}$;

(b) if $\lambda_{1}, \lambda_{2}$ are such that $\lambda^{\prime} \leqq \lambda_{1}<\lambda_{2} \leqq \lambda^{\prime \prime}$, then $\mathscr{A}\left(\lambda_{1}\right) \subset \mathscr{A}\left(\lambda_{2}\right)$;

(6.1) (c) if $\lambda_{0}$ is a value such that $\lambda^{\prime} \leqq \lambda_{0}<\lambda^{\prime \prime}$, then

$$
\mathscr{A}\left(\lambda_{0}\right)=\Pi \mathscr{A}(\lambda) \quad \lambda_{0}<\lambda \leqq \lambda^{\prime \prime} ;
$$

(d) if $\lambda_{0}$ is a value such that $\lambda^{\prime}<\lambda_{0} \leqq \lambda^{\prime \prime}$, then

$$
\mathscr{A}\left(\lambda_{0}\right)=\overline{\Sigma \mathscr{A}(\lambda)} \quad \lambda^{\prime} \leqq \lambda<\lambda_{0} .
$$

For each $\lambda, \lambda^{\prime} \leqq \lambda \leqq \lambda^{\prime \prime}$, the symbols $\iota(\lambda), \nu(\lambda)$ denote, respectively, the index and the nullity of $J$ on $\mathscr{A}(\lambda)$. Observe that the index $c$ is an integer valued function, monotone nondecreasing with increasing $\lambda$ on the interval. Moreover $\iota\left(\lambda^{\prime}\right)=0, \iota\left(\lambda^{\prime \prime}\right)=\iota_{a}$, the index of $J$ on $\mathscr{A}$. In general $\iota_{a} \neq 0$, so that there exist one or more values $\lambda_{0}$ (though but a finite number of such values) in the interval at which $\iota$ is discontinuous, with jump

$$
c\left(\lambda_{0}\right)=\iota\left(\lambda_{0}+\right)-\iota\left(\lambda_{0}-\right)>0 .
$$

Such a value $\lambda_{0}$ is called a focal point of $J$ relative to the family $\{\mathscr{A}(\lambda)\}$. The value $c\left(\lambda_{0}\right)$ is termed the order of $\lambda_{0}$ as a focal point. In virtue of property (d) in (6.1) the left hand limit $\iota\left(\lambda_{0}-\right)=\iota\left(\lambda_{0}\right)$, $\lambda^{\prime}<\lambda_{0} \leqq \lambda^{\prime \prime}$ (see [7], §16). The value $\iota\left(\lambda^{\prime \prime}+\right)$ is defined to be $\iota_{a}$. The following theorem, a restatement of results established in [7], is applied in the sequel.

TheORem 6.1. Let $\{\mathscr{A}(\lambda)\}$ be a family of subspaces of $\mathscr{A}$ having the properties (6.1). Then, for $\lambda^{\prime} \leqq \lambda<\lambda^{\prime \prime}$, the order $c(\lambda)$ of a focal point of $J$ relative to $\{\mathscr{A}(\lambda)\}$ is the dimension of the maximal submanifold $\mathscr{C}(\lambda)$ of $\mathscr{A}_{0}(\lambda)$ having the property that no nontrivial function in $\mathscr{C}(\lambda)$ is $J$-orthogonal to a subspace $\mathscr{A}\left(\lambda_{1}\right), \lambda_{1}>\lambda$. In the event the family $\{\mathscr{A}(\lambda)\}$ has the additional property that, whenever $\lambda_{1}, \lambda_{2}$ are values such that $\lambda^{\prime} \leqq \lambda_{1}<\lambda_{2} \leqq \lambda^{\prime \prime}$, there exists no nontivial function in $\mathscr{A}$ which is J-orthogonal to both $\mathscr{A}\left(\lambda_{1}\right)$ and $\mathscr{A}\left(\lambda_{2}\right)$, then $c\left(\lambda^{\prime}\right)=c\left(\lambda^{\prime \prime}\right)=0$, and for $\lambda^{\prime}<\lambda<\lambda^{\prime \prime}$, the order $c(\lambda)=$ $\nu(\lambda)$, the nullity of $J$ on $\mathscr{A}(\lambda)$.

7. Conjugate surfaces in $T$. Assume that there has been constructed a one parameter family $\{T(\lambda)\}$ of subsets of $T$, defined for $\lambda^{\prime} \leqq \lambda \leqq \lambda^{\prime \prime}$, having the following properties: 
(a) $T\left(\lambda^{\prime}\right)$ consists of a point of $\Omega$, or else has $m-1$ dimensional measure zero, while $T\left(\lambda^{\prime \prime}\right)=T$;

(b) $T(\lambda)$ is a region of class $B, \lambda^{\prime}<\lambda \leqq \lambda^{\prime \prime}$;

(c) if $\lambda_{1}, \lambda_{2}$ are such that $\lambda^{\prime \prime} \leqq \lambda_{1}<\lambda_{2}<\lambda^{\prime \prime}$, then $\overline{T\left(\lambda_{1}\right)} \subset \overline{T\left(\lambda_{2}\right)}$; and $T^{*}\left(\lambda_{1}\right) \cap T\left(\lambda_{2}\right)$ is not empty :

(7.1) (d) if $\lambda_{0}$ is value such that $\lambda^{\prime} \leqq \lambda_{0}<\lambda^{\prime \prime}$, then

$$
\overline{T\left(\lambda_{0}\right)}=\Pi \overline{T(\lambda)} \quad \lambda_{0}<\lambda \leqq \lambda^{\prime \prime} ;
$$

(e) if $\lambda_{0}$ is a value such that $\lambda^{\prime}<\lambda_{0} \leqq \lambda^{\prime \prime}$, then

$$
T\left(\lambda_{0}\right)=\Sigma T(\lambda) \quad \lambda^{\prime} \leqq \lambda<\lambda_{0} .
$$

Examples of families of sets which have these properties are given subsequently.

THEOREM 7.1. Let $\{T(\lambda)\}$ be a family of subsets of $T$ having properties (7.1). Define the family $\{\mathscr{A}(\lambda)\}$ of subsets of $\mathscr{A}$ as follows:

(i) $\mathscr{A}\left(\lambda^{\prime}\right)$ is the set whose sole member is the function which is identically zero on $T$, and $\mathscr{A}\left(\lambda^{\prime \prime}\right)=\mathscr{A}$;

(ii) If $\lambda$ is such that $\lambda^{\prime}<\lambda<\lambda^{\prime \prime}$, the $\mathscr{A}(\lambda)$ is the set of all $x \in \mathscr{A}$ having support set $\bar{S}_{x}$ contained in $\overline{T(\lambda)}$.

Then the family $\{\mathscr{A}(\lambda)\}$ is a family of subspaces of $\mathscr{A}$ for which the properties (6.1) hold.

Proof. Let $\lambda_{0}$ be a fixed but otherwise arbitrary value, $\lambda^{\prime}<\lambda_{0}<\lambda^{\prime \prime}$. It is readily verifiable that $\mathscr{A}\left(\lambda_{0}\right)$ is a closed linear manifold in $\mathscr{A}$. Let $\mathscr{H}\left(T\left(\lambda_{0}\right)\right), \mathscr{A}\left(T\left(\lambda_{0}\right)\right)$ refer to, respectively, the Hilbert space, and the subspace of $\mathscr{C}\left(T\left(\lambda_{0}\right)\right)$ composed of functions vanishing on $T^{*}\left(\lambda_{0}\right)$. Recall $\mathscr{A}\left(T\left(\lambda_{0}\right)\right)$ is characterized as being the subset of all $x \in \mathscr{C}\left(T\left(\lambda_{0}\right)\right)$ which when extended to vanish identically on $c\left(T\left(\lambda_{0}\right)\right)$, belong to $\mathscr{H}(\Omega)$. It follows that $\mathscr{A}\left(\lambda_{0}\right)$ is just the subspace $\mathscr{A}\left(T\left(\lambda_{0}\right)\right)$. For, if $x \in \mathscr{A}\left(\lambda_{0}\right)$, then $x$ restricted to $T\left(\lambda_{0}\right)$ belongs to $\mathscr{C}\left(T\left(\lambda_{0}\right)\right)$, while if $x$ is extended to $\Omega$ by setting $x(t)=0$ on $c\left(T\left(\lambda_{0}\right)\right)$, then $x \in \mathscr{H}(\Omega)$ since $x \in \mathscr{A}$. Thus $x \in \mathscr{A}\left(T\left(\lambda_{0}\right)\right)$. On the other hand

$$
\mathscr{A}\left(T\left(\lambda_{0}\right)\right)=\overline{C_{0}^{\infty}\left(T\left(\lambda_{0}\right)\right)} \subset \mathscr{A}\left(\lambda_{0}\right) \text {. }
$$

To show that property (c) of (6.1) holds, let $\lambda_{0}$ be fixed, $\lambda^{\prime} \leqq \lambda_{0}<\lambda^{\prime \prime}$. If $x \in \mathscr{A}\left(\lambda_{0}\right)$, then the support set

$$
\bar{S}_{x} \subset \overline{T\left(\lambda_{0}\right)} \subset \overline{T(\lambda)}
$$

whenever $\lambda_{0}<\lambda \leqq \lambda^{\prime \prime}$. Hence

$$
x \in \Pi \mathscr{A}(\lambda) \quad \lambda_{0}<\lambda \leqq \lambda^{\prime \prime} .
$$


On the other hand, suppose $x$ belongs to the intersection of the subspaces $\mathscr{A}(\lambda)$, for $\lambda_{0}<\lambda \leqq \lambda^{\prime \prime}$. Then whenever $\lambda_{0}<\lambda \leqq \lambda^{\prime \prime}, \bar{S}_{x} \subset \overline{T(\lambda)}$, so

$$
\bar{S}_{x} \subset \Pi \overline{T(\lambda)}=\overline{T\left(\lambda_{0}\right)}
$$

and hence $x \in \mathscr{A}\left(\lambda_{0}\right)$.

To establish property (d) of (6.1), let $\lambda_{0}$ be a value such that $\lambda^{\prime}<\lambda_{0} \leqq \lambda^{\prime \prime}$. Then it is clear from definitions that

$$
\Sigma \mathscr{A}(\lambda) \subset \mathscr{A}\left(\lambda_{0}\right)
$$

where the union is over all subspaces $\mathscr{A}(\lambda)$ for which $\lambda^{\prime} \leqq \lambda<\lambda_{0}$. On the other hand, as noted above,

$$
\mathscr{C}\left(\lambda_{0}\right)=\overline{C_{0}^{\infty}\left(T\left(\lambda_{0}\right)\right)} .
$$

Let $x \in C_{0}^{\infty}\left(T\left(\lambda_{0}\right)\right)$, so the support set $\bar{S}_{x} \subset T\left(\lambda_{0}\right)$. Now there must exist $\lambda_{x}, \lambda^{\prime}<\lambda_{x}<\lambda_{0}$, such that

$$
\bar{S}_{x} \subset T\left(\lambda_{x}\right) \subset T\left(\lambda_{0}\right) .
$$

For, if not, a sequence $\left\{\lambda_{p}\right\}$ exists such that $\lambda^{\prime}<\lambda_{p}<\lambda_{p+1}<\lambda_{0}, p=$ $1,2, \cdots$, and $\lambda_{p} \rightarrow \lambda_{0}$, and there exists a sequence $\left\{t_{p}\right\}$ of points such that $t_{p} \in \bar{S}_{x} \cap c\left(T\left(\lambda_{p}\right)\right), p=1,2, \cdots$, and $t_{p} \rightarrow t_{0} \in \bar{S}_{x}$. The sequence $\left\{t_{p}\right\}$ has the property that given a value $\lambda, \lambda^{\prime}<\lambda<\lambda_{0}$, there exists an integer $q_{\lambda}$ such that $t_{p} \in c(T(\lambda))$ whenever $p>q_{\lambda}$. Accordingly $t_{0} \in c(T(\lambda))$ for $\lambda^{\prime} \leqq \lambda<\lambda_{0}$, and hence

$$
t_{0} \in \Pi c\left(T(\lambda)=c(\Sigma T(\lambda))=c\left(T\left(\lambda_{0}\right)\right)\right.
$$

where the intersection and union are taken for $\lambda$ such that $\lambda^{\prime} \leqq \lambda<\lambda_{0}$. This is a contradiction in view of $t_{0} \in \bar{S}_{x}$, and $\bar{S}_{x} \subset T\left(\lambda_{0}\right)$. Thus a value $\lambda_{x}$ having the stated property must exist, and so $x \in \mathscr{A}\left(\lambda_{x}\right)$. Accordingly

$$
C_{0}^{\infty}\left(T\left(\lambda_{0}\right)\right) \subset \Sigma \mathscr{A}(\lambda)
$$

for $\lambda^{\prime} \leqq \lambda<\lambda_{0}$, and so

$$
\mathscr{A}\left(\lambda_{0}\right) \subset \overline{\Sigma \mathscr{A}(\lambda)} \subset \mathscr{A}\left(\lambda_{0}\right) .
$$

The following are examples of families of sets $\{T(\lambda)\}$ which have the properties (7.1).

Example 1. Let $t_{0} \in \Omega$ be fixed, and let $T(\lambda)$ denote the interior of the sphere $\left|t-t_{0}\right|=\lambda$, for $0<\lambda \leqq r, r$ a fixed positive number. Let $T(0)=\left\{t_{0}\right\}, T=\left\{t:\left|t-t_{0}\right|<r\right\}$.

EXAMPle 2. Let $T$ be a given interval $(a, b)$ having positive 
measure, and let $\lambda$ denote length measured along the diagonal joining the points $a$ and $b$, where $\lambda^{\prime \prime}=|b-a|$. Let $c_{k}$ denote the $k$-th direction cosine of the line joining $a$ to $b$. Let

$$
T(\lambda)=(a, a+\lambda c) \quad 0<\lambda \leqq \lambda^{\prime \prime}
$$

while $T(0)=\{a\}$.

EXAmple 3. Let $T=(a, b)$, and let $t_{0}$ denote the center of $T$. Define the family $\{T(\lambda)\}$ for $0<\lambda \leqq 1$ by

$$
T(\lambda)=\left(t_{0}-\frac{\lambda}{2}(b-a), t_{0}+\frac{\lambda}{2}(b-a)\right)
$$

and let $T(0)=\left\{t_{0}\right\}$.

EXAMPLE 4 . Let $S$ denote an interval $(a, b)$ of positive measure, and let $t_{0}$ be a point on the boundary $S^{*}$. Let $V$ denote a hypercube $\left(t_{0}-h / 2, t_{0}+h / 2\right)$, where $h>0$ is fixed. Let $T$ be the union of $S$ with $V$. Let $\{S(\lambda)\}$ be the family of expanding subsets constructed for the interval $S$ in the same manner as for the interval in Example 2 , for $0 \leqq \lambda \leqq \lambda^{\prime \prime}$, where $\lambda^{\prime \prime}=|b-a|$. Let $\{V(\lambda)\}$ be the family of cubes

$$
V(\lambda)=\left(t_{0}-\frac{\lambda h}{2}, t_{0}+\frac{\lambda h}{2}\right) \quad 0<\lambda \leqq 1
$$

centered about the point $t_{0}$, and let $V(0)=\left\{t_{0}\right\}$. Define the family $\{T(\lambda)\}$ of subsets of $T$ by

$$
\begin{aligned}
T(\lambda) & =S(\lambda) & & 0 \leqq \lambda \leqq \lambda^{\prime \prime} \\
& =S \cup V\left(\lambda-\lambda^{\prime \prime}\right) & & \lambda^{\prime \prime} \leqq \lambda \leqq \lambda^{\prime \prime}+1 .
\end{aligned}
$$

Instead of expanding to fill $S$ and then $T$, one can have the family of subsets $\{T(\lambda)\}$ expand to fill $V$ first, then $T$. Alternatively one can have the family $\{T(\lambda)\}$ expand into both sets simultaneously if

$$
T(0)=\left\{t_{0}\right\} \quad T(\lambda)=S(\lambda) \cup V\left(\lambda / \lambda^{\prime \prime}\right) \quad 0<\lambda \leqq \lambda^{\prime \prime} .
$$

In any case the desired properties (7.1) hold.

The following theorem shows that there exists a wide class of families $\{T(\lambda)\}$, each of which have the properties (7.1). The proof follows from the fact that closure and inclusion properties of sets is preserved under the transformations considered.

THeOREM 7.2. Let $T$ be a region of class $B$, and let $(T(\lambda))$ be a family of subsets of $T$ having the properties (7.1). Let $S$ be the 
image of $T, S(\lambda)$ the image of $T(\lambda), \lambda^{\prime} \leqq \lambda \leqq \lambda^{\prime \prime}$, under a continuous one-to-one transformation which is such that the transformation and its inverse satisfy a uniform Lipschitz condition on every compact subset of their respective domains. Then the family $\{S(\lambda)\}$ has the properties in (7.1) relative to the set $S$, which is a region of class $B$.

With the foregoing in mind one can relate the index theory given in [7] to the notion of conjugate surface for the Euler equation as follows. Assume that Eq. (5.2) has the following weak unique continuation property: if $T_{1}$ is a region of class $B, \bar{T}_{1} \subset T$, and if $x$ is a solution of the differential equation in $T$ which vanishes identically on $T-T_{1}$, then $x$ vanishes identically on $T$. Now let $u$ be a nontrivial solution of Eq. (5.2) on such a region $T_{1}$ and suppose $u$ vanishes on the boundary $T_{1}^{*}$. Let $y$ be the extension of $u$ to $T$ such that $y(t)=0$ on $T-T_{1}$. Then $y \in \mathscr{A}$, but $y \notin \mathscr{A} /$. For if $y$ is a $J$-null vector of $\mathscr{A}$, then $y$ is a solution of Eq. (5.2) on $T$. But then $y$ vanishes identically on $T$. Hence $u$ is identically zero on $T_{1}$, contrary to the supposition. Thus $y$ cannot be a $J$-null vector of $\mathscr{A}$. However, since $y$ restricted to $T_{1}$ is an extremal on $T_{1}$, it must be $J$-orthogonal to the subspace $\mathscr{A}\left(T_{1}\right)$. Observe $y$ is a $J$-null vector of $\mathscr{A}\left(T_{1}\right)$, but not of any subspace $\mathscr{A}(S)$ where $S \supset \bar{T}_{1}$.

The index $\iota_{a}$ of $J$ on $\mathscr{A}$ is given by the dimension of a maximal submanifold $\mathscr{C}$ of $\mathscr{A}$ on which $J(x) \leqq 0$ and which contains no nontrivial $J$-null vector of $\mathscr{A}$. In view of the preceding paragraph and the fact that $J(y)=0$ it is seen that $y$ belongs to such a submanifold $\mathscr{C}$. Thus $\iota_{a} \geqq 1$. A conjugate surface (of the Euler equation) is the boundary of a region of class $B$ on which there vanishes a nontrivial solution of the Euler equation. Accordingly $T_{1}^{*}$ is a conjugate surface. The existence of another conjugate surface $T_{2}^{*}$ distinct from $T_{1}^{*}$, where $T_{2} \supset T_{1}$, leads to the conclusion that the index $\iota_{a} \geqq 2$. These properties are analogous to those given for one dimensional problems in the calculus of variations.

The results given by Cordes ([5]) together with the smoothness assumptions stated in $\S 5$ for the coefficients and the strong Legendre condition imply that Eq. (5.2) has the weak unique continuation property. Another result of this type is given by Aronszajn ([1]) and Calderon ([2]).

Theorem 7.3. Assume the coefficients in Eq. (5.2) have the properties stated heretofore. Let $\{T(\lambda)\}$ be a family of subsets of $T$ having properties (7.1), and let $\{\mathscr{A}(\lambda)\}$ be the corresponding family of subspaces of $\mathscr{A}$ given by Theorem 7.1. Then

(a) a value $\lambda, \lambda^{\prime}<\lambda<\lambda^{\prime \prime}$, is a focal point of $J$ relative to the 
family $\{\mathscr{A}(\lambda)\}$ if, and only if, $T^{*}(\lambda)$ is a conjugate surface; moreover $\lambda^{\prime}$ and $\lambda^{\prime \prime}$ are not focal points;

(b) there are at most a finite number of conjugate surfaces in the family $\left\{T^{*}(\lambda)\right\}$;

(c) if $\lambda_{j}$ is a focal point, then the order $c\left(\lambda_{j}\right)$ is $\nu\left(\lambda_{j}\right)$, the nullity of $J$ on the subspace $\mathscr{A}\left(\lambda_{j}\right)$, and this is just the number of linearly independent solutions of Eq. (5.2) which vanish on $T^{*}\left(\lambda_{j}\right)$, in the maximal set of such nontrivial solutions;

(d) there exists a least focal point $\lambda_{1}>0$ in the interval $\lambda^{\prime}<\lambda<\lambda^{\prime \prime}$, so that for $\lambda^{\prime} \leqq \lambda<\lambda_{1}$ the index $\iota(\lambda)=0$ and $\nu(\lambda)=0$;

(e) let $\lambda_{1}, \cdots, \lambda_{N}$ be the focal points arranged in order of increasing magnitude, with respective orders $\nu\left(\lambda_{j}\right), i=1, \cdots, N$, then the index of $J$ on $\mathscr{A}$ is

$$
\iota_{a}=\sum_{j=1}^{N} \nu\left(\lambda_{j}\right)
$$

Proof. Since Eq. (5.2) has the requisite properties an argument exactly like that used above shows that whenever $\lambda_{1}, \lambda_{2}$ are values such that $\lambda^{\prime}<\lambda_{1}<\lambda_{2} \leqq \lambda^{\prime \prime}$, then there exists no nontrivial functions in $\mathscr{A}$ which are $J$-orthogonal to both $\mathscr{A}\left(\lambda_{1}\right)$ and $\mathscr{A}\left(\lambda_{2}\right)$. Hence by Theorem 6.1 the order of a focal point $\lambda_{j}$ is exactly the nullity $\nu\left(\lambda_{j}\right)$ of $J$ on $\mathscr{A}\left(\lambda_{j}\right)$. Clearly $\lambda^{\prime}$ is not a focal point. One sets $\iota\left(\lambda^{\prime \prime}+\right)=$ $\iota\left(\lambda^{\prime \prime}\right)$, so $\lambda^{\prime \prime}$ is not a focal point. There are but a finite number of focal points in the interval $\lambda^{\prime}<\lambda<\lambda^{\prime \prime}$. If $\lambda_{1}$ is the least, then $\nu(\lambda)=0$ for $\lambda^{\prime} \leqq \lambda<\lambda_{1}$, so $\iota(\lambda)=0$ on that subinterval.

It is noted that the index $\iota_{a}$ given by Eq. (7.2) is the same for every choice of a family $\{T(\lambda)\}$ of expanding subsets of $T$ having properties (7.1).

8. Oscillation and comparison theorem. The following theorem is a corollary of Theorem 7.1, [9]. It is observed that the proof does not depend on the weak unique continuation property assumed above for Eq. (5.2).

THEOREM 8.1. There exists an $\varepsilon>0$ such that if $S$ is a region of class $B, S \subset T$, with the diameter of $S$ at most $\varepsilon$, then $J(x)>0$ holds for all nontrivial $x \in \mathscr{A}(S)$. Accordingly there are no conjugate surfaces contained in $S$.

Proof. If $S$ is a region of class $B, S \subset T$, then each $x \in \mathscr{A}(S)$ is extended to vanish identically on $c(S)$, and $\mathscr{A}(S)$ is a subspace of A. Thus, in virtue of the theorem cited, there exists an $\varepsilon>0$ and an $h>0$ such that if $S \subset T$ and the diameter of $S$ is at most $\varepsilon$, then 
$J(x) \geqq h\|x\|^{2}$ holds on $\mathscr{A}(S)$. Suppose $S_{1}^{*}$ is a conjugate surface contained in $S$. Let $u$ be the corresponding nontrivial solution of the Euler equation on $S_{1}$ which vanishes on $S_{1}^{*}$. Let $y$ be the extension of $u$ which vanishes on $c\left(S_{1}\right)$. Then $y \in \mathscr{A}(S)$, and moreover

$$
J(y)=\int_{S}\left(\omega_{y} y+\omega_{\dot{y}_{i}} \dot{y}_{i}\right) d t=\int_{S_{1}}\left(\omega_{u} u+\omega_{\dot{u}_{i}} \dot{u}_{i}\right) d t=0 .
$$

Hence $y$ is the trivial function on $S$, and so $u$ is the trivial solution on $S_{1}$, contrary to the assumption. Thus there are no conjugate surfaces within $S$.

The following theorem is a consequence of Eq. (7.2) and the fact that if $J(x) \geqq 0$ holds on $\mathscr{A}$, then the index $\iota_{a}=0$.

THEOREM 8.2. If $J(x) \geqq 0$ holds on $\mathscr{A}$, then there are no conjugate surfaces properly contained in $T$.

COROLLARY 8.3. In addition to the assumptions of the strong Legendre condition and smoothness conditions made heretofore let $P(t)>0$ on $T$. Then no solution on $T$ of the differential equation

$$
\frac{\partial}{\partial t_{i}}\left(R_{i j} \frac{\partial x}{\partial t_{j}}\right)-P(t) x=0
$$

oscillates in $T$ in the sense that there exists no conjugate surface properly contained in $T$.

Theorem 8.4 is a consequence of Theorem 16.3 [7], and the discussion in $\S 7$.

THEOREM 8.4. Let

$$
J^{*}(x)=\int_{T}\left\{P^{*}(t) x^{2}+2 Q_{i}^{*}(t) x \dot{x}_{i}+R_{i j}^{*}(t) \dot{x}_{i} \dot{x}_{j}\right\} d t
$$

$(i, j=1,2, \cdots, m)$ be a quadratic form on $\mathscr{H}$ having suitable coeffcients $P^{*}(t), Q_{i}^{*}(t), R_{i j}^{*}(t)$ such that the properties of $J$ hold also for $J^{*}$. Moreover, suppose that

$$
J^{*}(x) \geqq J(x)
$$

holds for all vectors $x \in \mathscr{A}$. Let

$$
E^{*}(x)=\frac{\partial}{\partial t_{i}}\left(R_{i j}^{*} \frac{\partial x}{\partial t_{j}}\right)-x\left(P^{*}-\sum_{i=1}^{n} \frac{\partial Q_{i}^{*}}{\partial t_{j}}\right)=0
$$

be the Euler equation corresponding to $J^{*}$. Let $\{T(\lambda)\}$ be a family 
of subsets of $T$ having the properties (7.1). Then the theorems on focal points and conjugate surfaces hold for Eq. (8.1). Let $T_{1}^{*}$, $T_{2}{ }^{*}, \cdots, T_{N}^{*}$, be the distinct conjugate surfaces of $E q$. (5.2) ordered according to the increasing and distinct focal points of $J$ in the interval, and let $T_{1}^{* \prime}, T_{2}{ }^{* \prime}, \cdots, T_{N^{*}}^{* \prime}$ be the distinct conjugate surfaces of Eq. (8.1) ordered according to the increasing and distinct focal points of $J^{*}$ in the same interval. Let $T_{r}, r=1,2, \cdots, N$, be the member of the family $\{T(\lambda)\}$ having as its boundary $T_{r}^{*}$ and let $T_{r}^{\prime}, r=$ $1,2, \cdots, N^{*}$, be the member of the family $\{T(\lambda)\}$ having as its boundary $T_{r}^{* \prime}$. Then $T_{r} \subset T_{r}^{\prime}, r=1,2, \cdots, N^{*}$. If $J^{*}(x)>J(x)$ holds for all nontrivial functions $x \in \mathscr{A}$, then $\bar{T}_{r} \subset T_{r}^{\prime}, r=1,2, \cdots, N^{*}$.

Order relations between the conjugate surfaces stated in the conclusion of Theorem 8.4 hold for the conjugate surfaces of the differential equations

$$
\begin{gathered}
E(x)=\frac{\partial}{\partial t_{i}}\left(R_{i j} \frac{\partial x}{\partial t_{j}}\right)-P(t) x=0 \\
E^{*}(x)=\frac{\partial}{\partial t_{i}}\left(R_{i j}^{*} \frac{\partial x}{\partial t_{j}}\right)-P^{*}(t) x=0
\end{gathered}
$$

provided the operator $E$ is strongly elliptic and

$$
R_{i j}^{*}(t) \xi_{i} \xi_{j} \geqq R_{i j}(t) \xi_{i} \xi_{j} \quad t \in T
$$

holds for each $\xi \in \Omega$, and $P^{*}(t) \geqq P(t)$ and for each $t \in T$. If strict inequality holds for some $t \in T$ in at least one of these inequalities, then the proper inclusion of the conjugate surfaces $T_{r}^{*}$ in $T_{r}^{\prime}$ hold, for $r=1, \cdots, N^{*}$.

If, in Eq. (8.2), $P=P(t, \mu), \mu$ a real parameter, and is monotone strictly increasing with increasing $\mu$, for each $t \in T$ (for example if $\left.P(t ; \mu)=P_{1}(t)+\mu\right)$, then the proper inclusion of conjugate surfaces holds for the equations

$$
\begin{aligned}
& \frac{\partial}{\partial t_{i}}\left(R_{i j} \frac{\partial x}{\partial t_{j}}\right)-P(t ; \mu) x=0 \\
& \frac{\partial}{\partial t_{i}}\left(R_{i j} \frac{\partial x}{\partial t_{j}}\right)-P\left(t ; \mu^{*}\right) x=0
\end{aligned}
$$

where $\mu^{*}>\mu$.

9. Examples. In order to illustrate some of the results of the preceding sections the special case

$$
J(x)=\int_{T}\left\{\dot{x}_{i} \dot{x}_{i}-\mu x^{2}\right\} d t
$$


is considered, where $\mu$ denotes a constant. The corresponding Euler equation is

$$
\Delta x+\mu x=0 .
$$

As a first case let $T$ be the open interval $(0, b)$ in $\Omega$. The class of extremals is the class of solutions of Eq. (9.2). If $x$ is an extremal, then the $J$-orthogonality

$$
J(x, y)=\int_{0}^{b}\left\{\dot{x}_{i} \dot{y}_{i}-\mu x y\right\} d t=0
$$

holds for every $y \in \mathscr{A}$; moreover $x(t)$ is analytic on $(0, b)$.

The class $\mathscr{A}_{0}$ of $J$-null vectors of $\mathscr{A}$ consists of all solutions $x(t)$ of the problem

$$
\begin{aligned}
\Delta x+\mu x & =0 \quad \text { in } & & (0, b) \\
x & =0, & & t \in T^{*} .
\end{aligned}
$$

There are at most a finite number of linearly independent solutions of this problem. Separable solutions of Problem (9.3) are of the form

$$
x=\prod_{k=1}^{m} \sin \frac{n_{k} \pi t_{k}}{b_{k}},
$$

where the set $\left(n_{1}, \cdots, n_{m}\right)$ of positive integers satisfy the equation

$$
\sum_{j=1}^{m}\left(\frac{n_{j}}{b_{j}}\right)^{2}=\frac{\mu}{\pi^{2}}
$$

The set of functions of the form (9.4) spans the class $\mathscr{A}_{0}$ of $J$ null vectors of $\mathscr{A}$. There is but a finite number $\nu$ of linearly independent functions of this type, and the number $\nu$ is the nullity of $J$ on $\mathscr{A}$. For if $x$ is a function of the form (9.4) with positive integers satisfying (9.4), then $x \in \mathscr{A}_{0}$. Since the nullity of $J$ on $\mathscr{A}$ must be finite, there are at most a finite number of linearly independent functions of this type. Suppose now that $x \in \mathscr{A}_{0}$, and let the Fourier series for $x(t)$ in $T$ be

$$
x(t)=\sum_{p_{1}, \cdots, p_{m}=1}^{\infty} a_{p_{1} \cdots p_{m}} \prod_{k=1}^{m} \sin \frac{p_{k} \pi t_{k}}{b_{k}} .
$$

Since $x$ must satisfy Eq. (9.2),

$$
\sum_{p_{1}, \cdots, p_{m}=1}^{\infty} a_{p_{1} \cdots p_{m}}\left(-\sum_{j=1}^{m} \pi^{2}\left(\frac{p_{j}}{b_{j}}\right)+\mu\right) \prod_{k=1}^{m} \sin \frac{p_{k} \pi t_{k}}{b_{k}}=0,
$$

holds on every closed set in $T$. Hence whenever $a_{p_{1} \cdots p_{m}} \neq 0$, then the set $\left\{p_{j}\right\}$ of positive integers must satisfy 


$$
\sum_{j=1}^{m}\left(\frac{p_{j}}{b_{j}}\right)^{2}=\frac{\mu}{\pi^{2}} .
$$

There are but a finite number of distinct sets $\left\{p_{j}\right\}$ of positive integers which satisfy this last relation. Thus $x(t)$ must be a finite linear combination of functions of the type (9.4). The number $\nu$ of linearly independent functions of this type in a maximal set is the nullity of $J$ on $\mathscr{A}$. In fact the nullity of $J$ on $\mathscr{A}$ is given by $M$, where $M$ denotes the sum of the counts of all distinct sets $\left(p_{1}, \cdots, p_{m}\right)$ of positive integers which satisfy (9.5). A set $\left(p_{1}, \cdots, p_{m}\right)$ is counted $m ! / r$ ! times whenever it has $r$ of its elements alike.

Let $l$ denote the length of the diagonal from 0 to the point $b$. Define the family $\{T(\lambda)\}$ of subintervals by $T(\lambda)=(0, \lambda b / l), 0<\lambda<l$. Let $\{\mathscr{A}(\lambda)\}$ be the corresponding family of subspaces of $\mathscr{A}$. A function $x$ is a $J$-null vector of $\mathscr{A}(\lambda)$ if and only if $x$ is a linear combination of functions of the form

$$
y(t)=\prod_{k=1}^{m} \sin \frac{p_{k} \pi t_{k}}{c_{k}} \quad c_{k}=\frac{\lambda b_{k}}{l}, k=1, \cdots, m
$$

with $\left(p_{1}, \cdots, p_{m}\right)$ a set of positive integers satisfying

$$
\sum_{i=1}^{m}\left(\frac{p_{i}}{b_{i}}\right)^{2}=\frac{\lambda^{2} \mu}{\pi^{2} l^{2}}
$$

There is a set $\lambda_{1}, \cdots, \lambda_{N}$ of values $\lambda$ in the interval $0<\lambda<l$, such that for each $\lambda_{j}$ there exists at least one set $\left(p_{1}, \cdots, p_{m}\right)$ of positive integers satisfying (9.6). These values $\lambda_{j}$ of length along the diagonal are the distinct focal points of $J$ relative to the family $\{\mathscr{A}(\lambda)\}$. The corresponding intervals $\left(0, \lambda_{j} b / l\right)$ have boundaries which are the distinct conjugate surfaces $T^{*}\left(\lambda_{j}\right)$ of $J$ in $T$. Let $M\left(\lambda_{j}\right)$ denote the sum of the counts of sets $\left(p_{1}, \cdots, p_{m}\right)$ of positive integers satisfying (9.6) with $\lambda$ replaced by $\lambda_{j}$, the count being made as indicated previously, for $j=1, \cdots, N$. Then the index of $J$ on $\mathscr{A}$ is

$$
\iota_{a}=\sum_{j=1}^{N} M\left(\lambda_{j}\right)
$$

Consider now the equation

$$
\Delta x+P(t) x=0
$$

where $P \in C^{p-1}(T), p>m / 2+2$, and also bounded and integrable on $(0, b)$. Let $\lambda_{1}, \cdots, \lambda_{N}$ be the distinct focal points of $J$ relative to the family $\{T(\lambda)\}$ of subintervals of $T$. Suppose $P(t) \geqq \mu, t \in T$. Then there are values $\lambda_{1}^{\prime}, \lambda_{2}^{\prime}, \cdots, \lambda_{N^{\prime}}^{\prime}$ of lengths along the diagonal in $0<\lambda<l$, such that for each $\lambda_{j}^{\prime}$ there is at least one solution $x$ of Eq. (9.7) in 
the subinterval $\left(0, \lambda_{j}^{\prime} b / l\right)$ which vanishes on the boundary of the subinterval; moreover

$$
\left[0, \lambda_{j}^{\prime} b / l\right] \subset\left[0, \lambda_{j} b / l\right]
$$

If $P(t)>\mu$,

$$
\left[0, \lambda_{j}^{\prime} b / l\right] \subset\left(0, \lambda_{j} b / l\right)
$$

$j=1,2, \cdots, N$. Let $\nu\left(\lambda_{j}^{\prime}\right)$ denote the number of linearly independent solutions of Eq. (9.7) which vanish on the boundary of $\left(0, \lambda_{j}^{\prime} b / l\right), j=$ $1,2, \cdots, N^{\prime}$. Then

$$
\iota_{a}^{\prime}=\sum_{\jmath=1}^{N^{\prime}} \nu\left(\lambda_{\jmath}^{\prime}\right)>\sum_{\jmath=1}^{N} M\left(\lambda_{m}\right)=\iota_{a}
$$

where the numbers $M\left(\lambda_{j}\right)$ are those described in the preceding paragraph.

For a different example, let $T$ be the interior of the circle of radius $R$ about the origin, and let $m=2$. Separable solutions of Eq. (9.2) in polar coordinates which are single valued in $T$ are of the form

$$
x=J_{p}(\mu r)\left[c_{1} \cos p \theta+c_{2} \sin p \theta\right],
$$

where $c_{1}, c_{2}$ are constants, $p=0,1,2, \cdots$, and $J_{p}$ is the Bessel function of the first kind of order $p$. The class $\mathscr{A}_{0}$ of $J$-null vectors contains no nontrivial functions unless $\mu R>t_{01}$, where $t_{01}$ is the first zero of $J_{0}(t)$, and in any case the nullity will be either zero or one. Let $T(\lambda)$ be the interior of the circle of radius $\lambda$ about the origin, for $0 \leqq$ $\lambda \leqq R$. Then $T^{*}(\lambda)$ is a conjugate curve if and only if

$$
J_{p}(\mu \lambda)=0
$$

for some $p=0,1,2, \cdots$. Let $J_{0}, J_{1}, \cdots, J_{p}$ be the Bessel functions of integral order which have at least one zero in the interval $0<\lambda<\mu R$, and let $\nu_{q}$ be the number of zeros of $J_{q}(t)$ in this interval, for $q=$ $0,1, \cdots, p$. Then the index

$$
\iota_{a}=\sum_{q=0}^{p} \nu_{q} \cdot
$$

This value will be the same for any mode of expansion in sets $\{T(\lambda)\}$ having properties (7.1).

\section{REFERENCES}

1. N. Aronszajn, A unique continuation theorem for solutions of elliptic partial differential equations or inequalities of second order, J. Math. Pure Appl. (9) 36 (1957), 235-249.

2. A. Calderon, Uniqueness in the Cauchy problem for partial differential equations, 
Amer, J. Math. 80 (1958), 16-36.

3. J. W. Calkin, Functions of several variables and absolute continuity I, Duke Math. J. 6 (1940), 170.

4. A. B. Carson, An analogue of Green's theorem for multiple integral problems in the calculus of variations, Contributions to the Calculus of Variations, 1938-1941, University of Chicago Press.

5. H. O. Cordes, Über die eindeutige Bestimmtheit der Lösungen elliptischer differentialgleichungen durch Anfangsvorgaben, Akademie der Wissenschaften Göttingen, Math.- physikalische Klasse IIa. Nachrichten, 1956, 239.

6. K. Friedrichs, On the differentiability of the solutions of linear elliptic differential equations, Comm. Pure Appl. Math. 6 (1953), 299-326.

7. M. R. Hestenes, Applications of the theory of quadratic forms in Hilbert space to the calculus of variations, Pacific J. Math. 1 (1951), 525.

8. Calculus of variations and optimal control theory, J. Wiley and Sons, Inc., New York, 1966.

9. - Quadratic variational theory and linear elliptic partial differential equations, Trans. Amer. Math. Soc. 101 (1961), 306-350.

10. C. Morrey Jr., Functions of several variables and absolute continuity II, Duke Math. J. 6 (1940), 439.

11. Multiple integrals in the calculus of variations, Springer-Verlag, New York, 1966.

12. Multiple integral problems in the calculus of variations and related topics, Univ. of California Publ. in Math. 1 (1943).

Received August 26, 1968 and in revised form December 4, 1968. Part of a dissertation written in 1956 under the direction of Professor M. R. Hestenes, University of California, Los Angeles. The preparation of this paper was sponsored by the U. S. Army Research Office under Grant DA-31-124-ARO(D)-355. Reproduction in whole or in part is permitted for any purpose of the United States Government.

California State College, San Bernardino 


\section{PACIFIC JOURNAL OF MATHEMATICS}

\section{EDITORS}

H. ROYDEN

Stanford University

Stanford, California

\section{RichaRd PIERCE}

University of Washington Seattle, Washington 98105
J. DugundJI

Department of Mathematics

University of Southern California

Los Angeles, California 90007

BASIL GORDON

University of California

Los Angeles, California 90024

\section{ASSOCIATE EDITORS}
E. F. BECKENBACH
B. H. NeUMANN
F. WOLF
K. YOSHIDA

\section{SUPPORTING INSTITUTIONS}

UNIVERSITY OF BRITISH COLUMBIA CALIFORNIA INSTITUTE OF TECHNOLOGY

UNIVERSITY OF CALIFORNIA

MONTANA STATE UNIVERSITY

UNIVERSITY OF NEVADA

NEW MEXICO STATE UNIVERSITY

OREGON STATE UNIVERSITY

UNIVERSITY OF OREGON

OSAKA UNIVERSITY

UNIVERSITY OF SOUTHERN CALIFORNIA

\author{
STANFORD UNIVERSITY \\ UNIVERSITY OF TOKYO \\ UNIVERSITY OF UTAH \\ WASHINGTON STATE UNIVERSITY \\ UNIVERSITY OF WASHINGTON \\ AMERICAN MATHEMATICAL SOCIETY \\ CHEVRON RESEARCH CORPORATION \\ TRW SYSTEMS \\ NAVAL WEAPONS CENTER
}

The Supporting Institutions listed above contribute to the cost of publication of this Journal, but they are not owners or publishers and have no responsibility for its content or policies.

Mathematical papers intended for publication in the Pacific Journal of Mathematics should be in typed form or offset-reproduced, double spaced with large margins. Underline Greek letters in red, German in green, and script in blue. The first paragraph or two must be capable of being used separately as a synopsis of the entire paper. It should not contain references to the bibliography. Manuscripts, in duplicate if possible, may be sent to any one of the four editors. Please classify according to the scheme of Math. Rev. 36, 1539-1546. All other communications to the editors should be addressed to the managing editor, Richard Arens, University of California, Los Angeles, California, 90024.

50 reprints are provided free for each article; additional copies may be obtained at cost in multiples of 50 .

The Pacific Journal of Mathematics is published monthly. Effective with Volume 16 the price per volume (3 numbers) is $\$ 8.00$; single issues, $\$ 3.00$. Special price for current issues to individual faculty members of supporting institutions and to individual members of the American Mathematical Society: $\$ 4.00$ per volume; single issues $\$ 1.50$. Back numbers are available.

Subscriptions, orders for back numbers, and changes of address should be sent to Pacific Journal of Mathematics, 103 Highland Boulevard, Berkeley, California, 94708.

PUBLISHED BY PACIFIC JOURNAL OF MATHEMATICS, A NON-PROFIT CORPORATION

Printed at Kokusai Bunken Insatsusha (International Academic Printing Co., Ltd.), 7-17. Fujimi 2-chome, Chiyoda-ku, Tokyo, Japan. 


\section{Pacific Journal of Mathematics}

\section{Vol. 30, No. $3 \quad$ November, 1969}

Willard Ellis Baxter, Topological rings with property $(Y) \ldots \ldots \ldots \ldots . \ldots 5$

Sterling K. Berberian, Note on some spectral inequalities of $C . R$.

Putnam ..................................... 573

David Theodore Brown, Galois theory for Banach algebras . ........... 577

Dennis K. Burke and R. A. Stoltenberg, A note on p-spaces and Moore spaces ........................................ 601

Rafael Van Severen Chacon and Stephen Allan McGrath, Estimates of positive contractions....................................... 609

Rene Felix Dennemeyer, Conjugate surfaces for multiple integral problems in the calculus of variations ........................... 621

Edwin O. Elliott, Measures on countable product spaces.............. 639

John Moss Grover, Covering groups of groups of Lie type .............. 645

Charles Lemuel Hagopian, Concerning semi-local-connectedness and cutting in nonlocally connected continua .................. 657

Velmer B. Headley, A monotonicity principle for eigenvalues ........... 663

John Joseph Hutchinson, Intrinsic extensions of rings . . . . . . . . . . . . . 669

Harold H. Johnson, Determination of hyperbolicity by partial

prolongations .................................. 679

Tilla Weinstein, Holomorphic quadratic differentials on surfaces in $E^{3} \ldots 697$

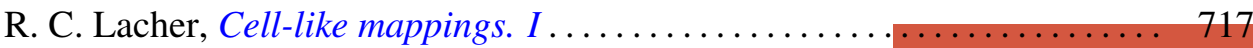

Roger McCann, A classification of centers

Curtis L. Outlaw, Mean value iteration of nonexpansive mappings in a

Banach space...

Allan C. Peterson, Distribution of zeros of solutions of a fourth order

differential equation.

Bhalchandra B. Phadke, Polyhedron inequality and strict convexity .. 765 Jack Wyndall Rogers Jr., On universal tree-like continua .

Edgar Andrews Rutter, Two characterizations of quasi-Frobenius rings

G. Sankaranarayanan and C. Suyambulingom, Some renewal theorems concerning a sequence of correlated random variables...

Joel E. Schneider, A note on the theory of primes........ . .

Richard Peter Stanley, Zero square rings .................

Edward D. Tymchatyn, The 2-cell as a partially ordered space

Craig A. Wood, On general Z.P.I.-rings................ 